\title{
EMIGRACIÓN Y CUIDADOS: AMBIGÜEDADES, CAMBIOS Y CONTINUIDADES DESDE LA PERSPECTIVA DE LAS ABUELAS CUIDADORAS EN COLOMBIA
}

\section{Emigration and care: Ambiguities, changes and continuities from Colombian grandmothers' point of view.}

\author{
Gisela M. Bianchi-Pernasilici*; Gioia Piras** \\ *Universidad del País Vasco/Euskal Herriko Unibertsitatea; ** Universidad del País Vasco/Euskal \\ Herriko Unibertsitatea \\ giselamarisa.bianchi@ehu.es; gioiapiras@gmail.com
}

\section{Palabras clave \\ Abuelas \\ Emigración \\ colombiana \\ Género \\ Organización del \\ cuidado}

\section{Keywords}

Grandmothers

Colombian

emigration

Gender

Care

organization

\section{Resumen}

En la actualidad, los aspectos relacionados con la organización de las tareas de cuidado de personas juegan un papel importante dentro del planteamiento y desarrollo de los viajes migratorios. Así, el propósito de este artículo es llevar a cabo una aproximación a las experiencias vividas por las abuelas colombianas que se quedan al cuidado de sus nietos y nietas tras la emigración de los progenitores a España. A través de entrevistas semiestructuradas realizadas en la región del Eje Cafetero en Colombia, nuestro objetivo es analizar el punto de vista de las abuelas en torno a la reorganización del cuidado a personas dependientes del hogar tras la emigración, haciendo hincapié en las emociones y sentimientos que la asunción del papel de cuidadora genera. Los resultados de nuestro estudio pretenden hacer una llamada de atención sobre los efectos emocionales que la migración familiar genera en las sociedades de origen, siendo éste un campo de investigación todavía poco explorado.

\section{Abstract}

Nowadays, care work plays an important role in order to understand current migrations flows. Thus, the aim of this article is to study the experiences of Colombian grandmothers who are taking care of their grandchildren after their parents' migration to Spain. By means of semistructured interviews realized in the Colombian region of Eje Cafetero, we analyzed the reorganization of care practices after the migration process from the grandmothers' point of view, focusing our attention on their feelings and emotions. The results of our study are a call of attention to the emotional effects that family migration process causes in the societies of origin, which constitutes a field still unexplored.

Bianchi-Pernasilici, G. y Piras, G., 2015, "Emigración y cuidados: Ambigüedades, cambios y continuidades desde la perspectiva de las abuelas cuidadoras en Colombia", en Papeles del CEIC, vol. 2015/2, no 132, CEIC (Centro de Estudios sobre la Identidad Colectiva), Universidad del País Vasco, http://dx.doi.org/10.1387/pceic.13472 


\section{INTRODUCCIÓN}

Los estudios sobre las migraciones internacionales se interesaron en sus comienzos principalmente por los cambios experimentados en las sociedades de destino y por los efectos de la movilidad en las vivencias de las personas migrantes, poniendo especial énfasis en los aspectos económicos, legales y laborales (Blanco, 1993; Colectivo IOE, 1999). No obstante, desde hace más de una década, la perspectiva transnacional aporta una manera más profunda y compleja de entender e investigar el fenómeno migratorio y las relaciones que se crean entre el "allí" y el "aquí". Es decir, se amplía la mirada al conjunto de transformaciones que ocurren en las sociedades tanto de origen como de destino (Blanco, 2007; Levitt, DeWind y Vertovec, 2003).

Ahora bien, buena parte de las investigaciones sobre los procesos transnacionales fijaron inicialmente su atención en cuestiones de tipo económico y político fundamentalmente (Delgado-Wise, Covarrubias y Rodríguez, 2004; Ostergaard-Nielsen, 2003), relegando a un plano secundario el estudio de otros ámbitos como la organización de los cuidados, que atañen en mayor medida a las experiencias y necesidades de las mujeres (Ariza, 2007; Hondagneu-Sotelo, 2007). En este sentido, el trabajo pionero de Arlie Hochschild pone de manifiesto la existencia de"cadenas mundiales de afecto y asistencia" entre los países de destino $y$ de origen de las migraciones, haciendo referencia a "una serie de vínculos personales entre gentes de todo el mundo, basadas en una labor remunerada o no remunerada de asistencia" (Hochschild, 2001: 188). A partir de este concepto, y conjuntamente con otras investigaciones (Hondagneu-Sotelo y Avila, 1997; Parreñas, 2001), la organización social de las labores de cuidado se convierte en un campo de estudio emblemático desde el que analizar las desigualdades de género tanto a escala global como local.

En este sentido, resulta interesante llevar a cabo una aproximación a las realidades subjetivas, especialmente de aquellas personas que no migran pero que cumplen papeles clave (Herrera, 2013), pues ayuda a comprender cómo se produce la migración, bajo qué circunstancias se hace posible y en qué medida afecta a la vida personal y familiar tanto de las personas que 'se van' como de las que 'se quedan' (D'Aubeterre, 2002; Marroni, 2000). En este contexto las abuelas desempeñan un papel emblemático; un caso poco estudiado (Yeates, 2005) y que a menudo 
representan el último, pero fundamental, eslabón de las 'cadenas globales de cuidado' (Molano-Mijangos, Robert y García Domínguez, 2012). Partiendo de esta mirada, el propósito del artículo es abordar la situación de las abuelas colombianas que se quedan en origen al cuidado de sus nietos/as tras la migración de los progenitores a España. Dos hipótesis constituyen la brújula del estudio: uno, las abuelas desempeñan un papel fundamental dentro de la transnacionalización de los cuidados y su consecuente reorganización en el seno de los hogares tras la emigración; y, dos, la emigración de sus hijas/os influye de manera ambivalente en las experiencias vitales de las abuelas, en sus relaciones familiares, sus emociones y sentimientos.

Además de la introducción, el texto se divide en tres apartados. El primero presenta una breve panorámica teórica sobre la relación entre los flujos migratorios y la organización transnacional de los cuidados. El segundo, explica las cuestiones metodológicas y las principales características del trabajo de campo. El tercero, da cuenta del análisis de los relatos de las abuelas en relación con las hipótesis formuladas. Y, por último, se exponen las cuestiones más relevantes del análisis a modo de conclusión.

\section{EL TRABAJO DE CUIDADOS EN CONTEXTOS TRANSNACIONALES}

\subsection{La relación entre el trabajo de cuidados y los flujos migratorios}

El estudio de la organización social de los cuidados en los hogares ha sido históricamente relegado a un segundo plano dentro de las Ciencias Sociales (Carrasco, Borderías y Torns, 2011). No obstante, Cristina Hughes (2002) considera que, en las últimas décadas, entender la manera en la que se desempeñan y se reparten las tareas de cuidados en la sociedad ha pasado desde la marginalidad al centro del debate social. De hecho, en la actualidad, el trabajo de cuidados es considerado un terreno de estudio emblemático para analizar las desigualdades de género, clase, etnia y generacionales, por lo que la autora lo coloca en la lista de los seis conceptos clave de los estudios de género.

Entendemos por trabajo de cuidados el conjunto de acciones cotidianas necesarias para el desarrollo y el bienestar de las personas, especialmente cuando son menores, ancianas y/o enfermas. Éste puede 
realizarse por los miembros de la familia o puede ser delegada a otras personas ajenas a ella. Si bien implica un trabajo material también se reconoce el aspecto afectivo y emocional que conlleva (Aguirre, GarcíaSaínz y Carrasco, 2005). Por ello, entendemos que este trabajo es parte de la reproducción de la especie humana pero ¿de qué manera se relaciona con los flujos migratorios?

En relación a los procesos que tienen lugar en los países destino, la situación actual puede definirse como de "crisis de cuidados" (Carrasquer, 2013; Pérez Orozco, 2006). Se utiliza este concepto para evidenciar que existen grandes dificultades para satisfacer una parte significativa de las necesidades de cuidado de la población a causa de cambios demográficos, sociales, económicos, políticos y de género ocurridos en las últimas décadas en las sociedades llamadas desarrolladas ${ }^{1}$. De esta manera, se pone de manifiesto la necesidad de replantear la actual organización social del cuidado, para repartir las tareas de manera más equitativa dentro de la sociedad y para asegurar cuidados adecuados a las personas dependientes. En este sentido, numerosos estudios evidencian el aumento de la demanda de servicios basados en asistencia a personas dependientes y de trabajos domésticos en los países ricos, mostrando su relación con los flujos migratorios de personas en un mundo cada vez más globalizado (Anderson, 2000; Parella, 2000; Sassen, 2003). En este sentido, Gioconda Herrera (2013) explica que en la década de los años sesenta y setenta del siglo pasado, los flujos migratorios eran protagonizados por trabajadores/as que alimentaban la producción industrial, mientras que actualmente lo están siendo por trabajadoras/es que se incorporan masivamente en el mercado laboral de servicios, especialmente en el sector doméstico y de cuidados.

Si hablamos del contexto de origen, Juliana Martínez Franzoni (2008) argumenta que en América Latina las políticas neoliberales han conllevado un debilitamiento de los Estados del Bienestar que, a su vez, ha producido una significativa estratificación dentro de la organización del cuidado. Es decir, según la clase, la etnia, el género y la posición que

\footnotetext{
'La bibliografía experta hace referencia principalmente a tres cambios: (1) la "revolución reproductiva" (Maclnnes y Pérez Díaz, 2008); (2) las variaciones en las concepciones, prácticas y calidad de los cuidados, como los cambios en las formas familiares y de roles, expectativas e identidades de género; (3) la reducción significativa de la cobertura del Estado del Bienestar para los servicios sociales y de cuidados (Gálvez y Torres, 2010).
} 
se tiene en la estructura social, se enfrentan de manera diferente las necesidades de la reproducción del hogar y los riesgos a los que las personas y las familias se enfrentan cotidianamente. En este contexto, Gioconda Herrera (2012) explica que la migración se convierte en una de las estrategias para enfrentar los diferentes riesgos a los que las familias están expuestas. Para muchas personas en los países del sur global las diferencias de salarios en todo el mundo ofrecen un poderoso incentivo para la movilidad internacional. Tener acceso a una diversidad de empleos, e incluso a una variedad de economías nacionales (UE), puede ser un seguro contra las inestabilidades que la propia globalización genera, especialmente en los países empobrecidos (Hochschild, 2001). En este sentido, como defiende Ana María Arteaga (2010), unas de las principales causas de la migración andina, y en este caso de la migración colombiana, son las crisis económicas y la falta de oportunidades laborales en el país de origen. La privatización de los servicios antes públicos, como el sanitario y el educativo, junto a los recortes en el gasto público, dejan la satisfacción de las necesidades de cuidados en el ámbito privado y familiar, obligando a las mujeres -en muchos casos jefas de hogar- a buscar soluciones económicas a través de su inserción en el mercado laboral informal (en muchas ocasiones en el trabajo de cuidados), tanto en los países de origen como en los de destino de la migración (Gil Araújo, 2005; Sørensen, 2008; Echeverri Buriticá, 2014). Por ello no es de extrañar que la emigración colombiana, así como la de otros países andinos, ha sido liderada por mujeres (Pedone, 2006, Bianchi-Pernasilici, 2014), llegando a ser para el caso de España una inmigración altamente feminizada y familiar, y que conlleva reconfiguraciones de género y generacionales dentro de la organización social y familiar (Echeverri Buriticá, 2014).

En definitiva, las realidades de destino y de origen están conectadas a través de las experiencias de vida de las personas migrantes y de las que se quedan $y$, en este contexto, se pone de manifiesto que las labores de cuidado, la reproducción de la familia y de los seres humanos, juegan un papel importante en la determinación de las causas y los efectos de los flujos migratorios. Algunas autoras señalan cómo en los países de origen las migraciones producen la acentuación de las desigualdades de género, clase y origen de las personas (Escrivá, 2004; Zimmerman, Litt y Bose, 2006) y, por este motivo, en los últimos años se ha intensificado el 
debate sobre la reproducción social y la internacionalización de los trabajos de cuidados dentro y fuera de la academia (Pérez Orozco, 2006).

La relación entre los flujos migratorios y la reorganización de los cuidados en origen y en destino, está igualmente produciendo, y a su vez retroalimentando, un contexto que Mary K. Zimmerman, Jacquelyn S. Litt y Christine E. Bose (2006) llaman de "múltiples crisis de cuidados" y que se caracteriza por cuatro elementos. Primero, aunque las condiciones difieren, tanto en los países de origen de la migración como en los de destino, existen demandas y necesidades de cuidados que están siendo difíciles de satisfacer. Así, para el caso de los países latinoamericanos, predomina la necesidad de cuidados en la esfera de la crianza de la población infantil, mientras que para Europa y en concreto España predomina la demanda de cuidado para la población de edad avanzada (Durán, 2011). Segundo, la mercantilización de los cuidados está generando nichos laborales caracterizados por la precariedad y la explotación laboral. Tercero, las posibilidades, servicios, herramientas y prácticas en la reproducción social se ven influidas por la lógica neoliberal y la consecuente reorganización del Estado del Bienestar. Y, cuarto, se produce la creación o reproducción de sistemas de estratificación basados en la etnia, la clase, el género y la edad como consecuencia de la globalización de los trabajos de cuidados.

Asimismo, es necesario destacar el interés de analizar estas situaciones en un momento histórico marcado por la crisis económica en los países de destino de las migraciones. En concreto para el caso estudiado, España, los efectos de la crisis sobre el mercado laboral han sido intensos, aún más para la población extranjera. Según los datos de la Encuesta de Población Activa del INE, las tasas de paro en referencia a este colectivo de personas ascienden notablemente, pasando de ser el $10,1 \%$ para hombres y $15,1 \%$ para las mujeres en 2006 , al $39,2 \%$ y $34,2 \%$, respectivamente, en 2012. Tener en cuenta esta situación es fundamental para entender los discursos de las abuelas colombianas sobre los efectos de la emigración en sus vidas. Así, se lleva a cabo una aproximación a las consecuencias transnacionales de la recesión económica sobre las vidas de las abuelas, al ser justamente esas situaciones críticas las que ponen de relieve cómo la organización del cuidado depende de un "delicado engranaje" entre una función y otra, 
entre una persona y otra y donde la falla de un lado afecta inevitablemente toda la cadena (Anderson, 2007).

\subsection{Emociones, circulación de cuidados y hogares transnacionales}

Como argumenta María Jesús Izquierdo (2003), las tareas de cuidados son muy diversas puesto que cada persona tiene su manera de entenderlas y desarrollarlas. Éstas implican actos pequeños y sutiles, conscientes e inconscientes, en los que intervienen sentimientos, acciones, conocimientos y tiempo. Mientras se realizan entra en juego, por una parte, la manera en que la persona cuidadora realiza estas labores, por otra, la manera en que viene percibido el cuidado brindado $y$, por último, la relación que se establece entre las dos personas: la que brinda y la que recibe los cuidados. Las tareas de cuidados pueden, de hecho, ser una arma de doble filo al poder generar sentimientos ambivalentes de gratitud y resentimiento, deuda y lealtad, autocomplacencia y altruismo, "en definitiva, poder y explotación" (Vega Solís, 2009: 97). De esta forma, cada mujer vive de manera particular la tensión entre el deseo de cuidar y el deseo de no cuidar, el tiempo para el cuidado de las otras personas y el tiempo para el autocuidado.

Por ello, resulta interesante estudiar la situación de las personas cuidadoras desde una perspectiva sociológica para profundizar los conocimientos sobre los mecanismos y contextos sociales que generan tensiones y percepciones ambivalentes (Merton, 1980) y que quedan incorporados en las experiencias de las personas. En este sentido, las tareas de cuidado, generan estados de ánimo ambivalentes que navegan entre las emociones y los sentimientos. El contacto físico con otras personas y las relaciones de intimidad que se establecen provocan sensaciones definidas como emociones -caracterizadas por ser inmediatas, involuntarias e inevitables-, tales como la angustia, la ternura, la alegría, la ansiedad etc. A su vez las sensaciones, con el paso del tiempo y el asentamiento de su experimentación pueden transformarse en sentimientos. Éstos se clasifican como la traducción socializada de la emoción o del estado emocional sentido (Cerulo, 2009). Tanto las emociones como los sentimientos están determinados por el contexto social y cultural, el cual enmarca lo que debemos o no debemos sentir emocionalmente, cuando y como sentirlo o reprimirlo. 
La subjetividad emocional de cada persona está constituida por un conjunto de normas emocionales que indican intensidad, dirección y duración del sentimiento (Hochschild, 1975; Bericat, 2000). Al ser la migración un hecho social total, es decir un hecho que engloba todas las esferas del ser humano (Mauss, 1929; Sayad 1999), parece fundamental tener en cuenta los efectos emocionales que se desencadenan en su regazo. En este sentido, a causa de la emigración de uno de sus miembros se producen notables transformaciones en las unidades familiares de origen $y$, sobre todo cuando los cambios afectan a las tareas de cuidados, se pueden generar sentimientos encontrados.

Es precisamente en este aspecto en el que nos queremos detener: en el ejercicio del cuidado en el lugar de origen por parte de las personas responsables, en este caso las abuelas, y los efectos que ello tiene sobre sus vidas. Con la emigración, el ejercicio del cuidado se traslada a otras personas de la familia a través de acuerdos internos (González y Restrepo, 2010; Bianchi-Pernasilici, González-Rábago y Piras, 2015), y la elección sobre en quién delegar el cuidado de la descendencia suele basarse en el mandato de género (Izquierdo, 2003), así como en la experiencia de las personas, recayendo, en muchos casos, en las abuelas. En este sentido, como señalan algunos estudios (Pérez Ortiz, 2006; Villalba Quesada, 2002), la cuestión de las abuelas cuidadoras es un fenómeno compartido tanto en destino como en origen, en familias migrantes y en familias no migrantes, no obstante, es un campo de estudio en el que aún hay que profundizar. Familias de migrantes, hogares transnacionales y la consiguiente "circulación de los cuidados" (Baldassar y Merla, 2014) están siendo investigados sobre todo desde el contexto de destino. Por estas razones, siendo las abuelas cuidadoras uno de los eslabones todavía invisibilizado de las cadenas mundiales de cuidados y afectos, nos parece importante centrarnos exclusivamente en sus subjetividades y experiencias.

Los hogares que se desarrollan en un contexto transnacional se caracterizan por la mescolanza de los cuidados: por una parte, los cuidados delegados a personas que se quedan en origen $y$, por otra, cuidados realizados a través de la distancia. Esto no significa, sin embargo, que todas las familias separadas geográficamente constituyan hogares transnacionales, siendo necesario para ello, como apuntan Deborah F. Bryceson y Ulla Vuorela (2002), un fuerte sentido de unidad 
en su concepción del bienestar colectivo y una interiorización de los vínculos entre sus miembros. A pesar de la frecuente mezcolanza de causas y efectos que rodea al estudio de la familia transnacional y de los cuidados, parece que factores como la edad de las criaturas que se quedan en origen, qué progenitor es el emigrante, la involucración de la prole en el proyecto migratorio de los adultos, el ejercicio de afectividad en la distancia, las condiciones familiares antes de la migración, o la atención en el cuidado y las emociones en la cercanía de los cuidadores, tienen gran incidencia (Wagner, 2008; Lagomarsino, 2014).

\section{Metodología y PeRfil de LA POBlación ENTREVISTAdA}

Los resultados que se presentan a lo largo de este artículo proceden de una investigación más amplia llevada a cabo entre 2010 y 2012 bajo el título "Implicaciones sociales de las migraciones transnacionales: más allá de las remesas. El caso del sistema migratorio entre países andinos y España"2. A nivel metodológico, el estudio se basó en la aplicación de técnicas tanto cuantitativas como cualitativas dirigidas a varios colectivos de la población andina, pero, en este artículo hacemos referencia únicamente a las entrevistas semi-estructuradas realizadas al grupo de abuelas colombianas.

El trabajo de campo se realizó entre enero y febrero de 2012 en la región del Eje Cafetero de Colombia. La elección del lugar para realizar el trabajo de campo se basó en la intensidad del flujo migratorio desde esta región a España, que sitúa un sistema migratorio específico y con características singulares. A partir de la década de los noventa, España se ha ido consolidando como el principal destino de la migración internacional originaria del Eje Cafetero (Mejía et al., 2009). La intensidad del fenómeno migratorio en esta zona es una de las más altas del país, con porcentajes de hogares con experiencia migratoria internacional superiores al 14\% (Garay y Rodríguez, 2005). Por estas razones el trabajo de campo se ha realizado en esta zona del país, puesto que, en los municipios nombrados se prevé una mayor intensidad en sus efectos no solo a nivel individual y familiar, sino también a nivel social y

\footnotetext{
${ }^{2}$ Investigación desarrollada por XENIA, Grupo de investigación sobre migraciones, alteridad y desarrollo humano de la Facultad de Ciencias Sociales y de la Comunicación, UPV/EHU. Este proyecto, con referencia CSO2009-08469 (subprograma SOCI), ha sido financiado por el Plan Nacional de I+D+i (2010-2012) del Ministerio de Ciencia e Innovación.
} 
comunitario, y de esta manera el contexto resulta adecuado para dar cuenta de los efectos de la emigración de personas al extranjero, dejando a sus hijos/as a cargo de sus ascendientes, en este caso las abuelas.

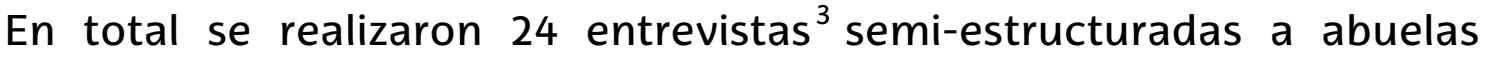
cuidadoras de sus nietos y nietas, cuyos padres/madres (o uno de ellos) residen en España. La edad de las entrevistadas varía entre los 50 y los 80 años, y la edad de las criaturas a su cargo entre los 5 y los 19 años. Las mujeres entrevistadas, salvo en algunos pocos casos, viven con sus maridos o con algunos de sus otros hijos/as no emigrados, además de con los nietos/as a cargo. Por último es significativo constatar que en su gran mayoría las entrevistadas son las responsables principales de los hijos/as de sus propias hijas mujeres que han emigrado ${ }^{4}$.

El análisis del material empírico se realizó a través de cuatro preguntas de investigación imbricadas con las dos hipótesis de partida. En relación con la primera hipótesis - el papel fundamental de las abuelas en la transnacionalización de los cuidados-, nos plantemos: (1) ¿cuál es la valoración general que hacen las abuelas de la emigración en tiempo de crisis?; y, (2) ¿cuál es el papel de las abuelas en la reorganización del ejercicio del cuidado tras la emigración? Y, en relación con la segunda hipótesis -la ambivalencia en las experiencias vitales de las abuelas a raíz de la migración de sus hijos-, los interrogantes son: (3) ¿de qué manera la emigración afecta a sus relaciones familiares, en este caso: con la persona migrante y con los nietos/as en origen a su cargo; y, (4) ¿cuáles son las reflexiones sobre su propia situación y sobre sus emociones y sentimientos al respecto?

\footnotetext{
${ }^{3}$ Las entrevistadas han sido contactadas a través del método de bola de nieve a partir de los datos proporcionados por algunas instituciones públicas municipales como centros educativos o de atención a la familia.

${ }^{4}$ Sólo en dos de los casos es el hijo varón quien ha emigrado y en cuatro más se trata de sus nueras o yernos.
} 
4. LA RESPONSABILIDAD DEL CUIDADO Y SU REORGANIZACIÓN TRAS LA EMIGRACIÓN. UNA MIRADA DESDE LA PERSPECTIVA DE LAS ABUELAS CON NIETOS/AS A CARGO EN ORIGEN

\subsection{Las valoraciones sobre la emigración y los cambios derivados de la crisis económica}

Como se evidencia en otros estudios citados anteriormente (Herrera y Carrillo, 2009), la opinión sobre el hecho migratorio de aquellas personas que lo experimentan de forma personal pero indirecta - a causa de la marcha de un familiar cercano- se ve influenciada por la vivencia de los cambios a nivel familiar e individual. Por ello, para verificar la primera hipótesis, y para tener una visión general de las consecuencias del fenómeno migratorio en la vida de las personas entrevistadas, se toma en cuenta la valoración que ellas hacen de la emigración de su prole hacia España.

A través del análisis de los relatos de las abuelas, se detecta una idea general: la bondad de la emigración cuando ésta supone un beneficio económico, que a menudo es concebido únicamente en términos familiares. En este sentido, existe un discurso casi unánime acerca de la falta de oportunidades en Colombia y de la búsqueda de un mejor futuro para la familia como las razones fundamentales que llevan a la emigración (Arteaga, 2010). Ahora bien, se produce una valoración ambivalente sobre el fenómeno migratorio, poniendo de manifiesto la tensión que se percibe entre los beneficios económicos y las pérdidas afectivas generada por la lejanía, es decir entre "la importancia del dinero" y "la importancia de los afectos". Esta tensión de nivel microsocial, planteada en varias ocasiones por las abuelas, se relaciona con lo que desde la economía feminista se conceptualiza como la tensión entre la "sostenibilidad de la vida" y la "acumulación de capital" en las sociedades capitalistas (Pérez Orozco y Lafuente, 2013).

En este sentido, con la situación de crisis económica de los principales países receptores de migración, y en concreto en el caso español (Gálvez y Torres, 2010; Colectivo IOÉ, 2012), la ambivalencia entre el beneficio económico y la pérdida afectiva se hace aún más patente. Es decir, en aquellas familias cuyos migrantes están siendo afectados por la crisis económica en España, la balanza se inclina del lado de la pérdida. Por una parte, las abuelas subrayan la compensación material que suponía la emigración cuando la situación económica en el país de destino permitía un importante envío de remesas a la familia en origen pero, por otra parte, expresan el impacto que ha tenido la crisis económica para ellas y las familias que las recibían. Así, desde una perspectiva 
transnacional, se aprecia la manera en la que los efectos de la crisis de los países de destino de las migraciones se extienden a nivel global, cruzando las fronteras estatales y teniendo consecuencias nefastas a miles de kilómetros de distancia de dónde se origina, a causa de las dependencias económicas creadas por las remesas.

Un elemento reiterado en el discurso de las abuelas es el deseo de retorno a Colombia de aquellos migrantes que están sufriendo la crisis económica en España. Sin embargo, dicho deseo no siempre es posible cumplirlo por la precaria situación económica que viven las personas inmigrantes, que llegan a sentirse atrapadas por la situación económica:

En el caso de mis hijos porque yo tengo dos allá, se fueron con la ilusión de tener algo, de salir adelante, de prosperar, pero fue una mentira muy grande. En este momento sinceramente no se han devuelto porque no tienen ni para el pasaje si quiera (E11: abuela María ${ }^{5}$, Santuario).

\subsection{El rol de las abuelas: el ejercicio del cuidado entre el cambio y la continuidad}

Los relatos de las abuelas colombianas permiten detectar que su protagonismo en el ejercicio de cuidado hacia sus nietos/as antes de la emigración tiene un peso muy importante en la toma de decisión de emprender o no el viaje migratorio. Según ellas, las personas que cuentan con una red de apoyo familiar para la satisfacción de las necesidades de cuidado de sus menores, eligen con más facilidad que otras el camino de la movilidad internacional como una opción plausible para llevar adelante la reproducción de su hogar.

En muchos de los casos analizados, la labor de cuidado de las abuelas hacia sus nietos/as arranca antes de la migración internacional del progenitor, a veces incluso desde el mismo nacimiento de los menores, debido a la obligación de las madres/padres de ausentarse de la casa por cuestiones de trabajo. Así las cosas, se pone de manifiesto la existencia de situaciones donde las necesidades cotidianas de cuidados se convierten en difíciles de resolver en los países de origen (Zimmerman et al., 2006), y donde las abuelas parecen desempeñar un papel emblemático antes y después del proceso migratorio. En este sentido, y de manera análoga a lo que señalan algunas autoras en referencia a otros contextos migratorios, se deduce que también en el caso de las abuelas colombianas existe una relación entre el hecho de haber

\footnotetext{
${ }^{5}$ Todos los nombres de las entrevistadas han sido modificados por otros inventados, respetando así la confidencialidad de sus testimonios.
} 
ejercido esta labor previamente a la emigración y el desarrollo de la migración en sí misma.

El hecho de poder delegar el cuidado de las criaturas a las abuelas, bien sean maternas o paternas, en muchos casos reside en la continuidad de la residencia. Es decir, en las situaciones analizadas, tanto cuando se trata de madres solteras como de parejas, los progenitores ya compartían techo con sus madres (abuelas) para satisfacer las necesidades de cuidado. Por consiguiente, se identifica que, en algunas situaciones, la partida al extranjero no ha sido causa de grandes cambios en la estructura familiar, puesto que las criaturas ya convivian con ellas anteriormente:

Por lo general siempre han vivido en compañía de nosotros, entonces al irse la mamá tengo más responsabilidad, tengo que estar más pendiente, pero normal, yo siempre lo he hecho (E7: abuela Rita, Santuario).

El cuidado de las/los menores recae principalmente en las abuelas, no obstante, cabe señalar que también existen redes familiares de apoyo que participan en el desempeño de estas tareas. Las situaciones varian dependiendo de las necesidades de los niños/as y de las mejores o peores condiciones de la abuela para realizar las labores diarias que demandan las criaturas. En los casos estudiados, las redes familiares toman parte especialmente de tareas relacionadas con el desarrollo escolar - el cumplimiento de los deberes en la casa- y la comunicación entre familia y escuela. De esta manera, se observa cómo entran en juego otros parientes, por ejemplo abuelos y tías/os. Estas redes, no sólo participan en la atención de tipo material, sino que colaboran igualmente en el aspecto del cuidado más relacionado con lo afectivo y lo emocional:

El niño siempre se ha criado en este ambiente, entre el tío-papa y mi persona. (...) Nosotros le hemos enseñado a querer a la mamá, a respetarla, y sus sentimientos lógico, pero es un amor que él sabe que ahí está pero que no es como nosotros que estamos constantemente con él. En ese punto no le ha hecho falta nada, ni disciplina, ni cuidado, ni amor, nada de esas cosas (E11: abuela María, Santuario)

Al analizar el material empírico llama la atención la ausencia total o parcial de la figura paterna (Chant, 1992). Es decir, cuando el padre no ha emigrado al extranjero, esta figura no tiene protagonismo en las redes familiares de apoyo en el cuidado de las criaturas. Dos situaciones son las más recurrentes dentro de los casos estudiados: por un lado, aquella en la que el padre nunca ha ejercido como tal o ha abandonado el hogar, 
y con ello su rol, hace mucho tiempo; $y$, por otro lado, aquella en la que el padre está presente pero el ejercicio del cuidado es de baja intensidad o muy esporádico.

A partir de los relatos se constata que los aspectos relacionados con la organización del cuidado de las personas dependientes, en este caso de las criaturas dejadas en origen, es un factor clave a la hora de plantear y desarrollar un proyecto migratorio y formar un hogar transnacional. En este sentido, las abuelas cumplen con un rol fundamental, no sólo en la gestión y ejercicio de los cuidados, sino también a menudo con la tarea de mantener vivos esos vínculos de los que hablan Deborah F. Bryceson y Ulla Vuorela (2002) entre los componentes de la familia para que se sientan parte de una unidad, alimentando la cohesión del hogar y recordando la presencia de las figuras materna y/o paterna en la ausencia.

\subsection{Cambios en las relaciones interpersonales en la familia tras la emigración}

Atendiendo a los cambios detectados por las abuelas sobre su relación con las personas emigradas y con nietos/as, se percibe una cierta discordancia según el ámbito al que se hace referencia: el afectivo y/o el de la relación cotidiana. Ambas relaciones están caracterizadas por la ambivalencia.

Respecto a las relaciones entre madre (abuela cuidadora) y descendiente emigrada/o, se percibe que el proceso migratorio afecta al plano cotidiano, pero no tanto al afectivo. Por lo general, dicen que las relaciones afectivas no se ven perturbadas por la migración, mientras que si se habla del ámbito cotidiano, existe una verbalización explícita por parte de las abuelas sobre la manera en la que las relaciones se ven afectadas en la distancia. Como también se señala en otros trabajos (Puyana, 2009), se destaca que el vínculo con su hija/o migrante se ve confinado a relaciones de tipo informativo sobre cuestiones clave como la salud, la escuela de los menores o cuestiones prácticas como el envío de las remesas, y que, además, es mediada por aparatos como el teléfono o el ordenador, la webcam y las fotos.

Respecto a la relación que se establece entre la abuela cuidadora y las/os descendientes de la persona emigrada, entre los factores que entran en juego, la edad de los menores en el momento de la migración parece ser muy importante (Wagner, 2008). Muchas veces estas relaciones se encuentran marcadas por la dependencia emocional. En los casos en los que los menores quedaron a cargo de la abuela desde muy pequeños, incluso desde recién nacidos, la sustitución de la figura 
materna-paterna se produce de forma tanto consciente como inconsciente. El tratamiento de éstos como si fueran sus propios hijos/as es una constante en el discurso de las cuidadoras entrevistadas:

Bien me siento feliz como que estuviera levantando mis hijos, si porque son como mis hijos, porque yo desde pequeñitos los he tenido, los vi nacer, estoy al pie de ellos y me siento feliz con ellos (E8: abuela Tere, Santuario).

Además, como señalan Rosario Aguirre,Cristina Saínz y Cristina Carrasco (2005) el desempeño de las tareas de cuidado crea relaciones bidireccionales entre las personas $y$, como se desprende del análisis de las entrevistas, dicha dependencia no es unidireccional entre abuelas y nietos/as, sino también puede que a menudo se produzca a la inversa, mostrando el apego de muchos de ellos y ellas hacia quienes han ejercido como sus cuidadoras principales desde la infancia y/o durante una gran parte de su vida. De tal manera, ante la cuestión de una posible reagrupación familiar de los menores en destino, una parte importante de las entrevistadas alude a que las y los niños/as no desean irse a vivir con los progenitores por no querer la separación de la persona que ha ejercido como madre-padre durante un largo periodo.

No, ella dice que llevársela por allá, la niña no le gusta, y que tal vez yo aquí sola, y que la niña no es capaz de dejarme sola porque yo la he cuidado mucho y que ella no es capaz de quitarme la niña, (...). No pues, bien y todo ella [la nieta] me dijo: mamita pues usted me reemplaza a mamá también, porque usted me cuidó desde muy chiquitica... Si, ella me cuenta todo, que me siente como una amiga y como la mamá también, me dice que soy la mamá (E27: abuela Adelinda, Pereira).

Desde el punto de vista de las abuelas, el sentimiento de ausencia tiene igualmente una incidencia muy significativa en la vivencia de las y los nietos, especialmente si se trata de la emigración emprendida por mujeres madres argumentándolo con expresiones como: "la mamá es algo irremplazable". Como afirma Heike Wagner (2008), en los discursos sociales en los países de origen, dicha ausencia se relaciona de manera casi automática con efectos negativos, en relación al comportamiento del menor y en la aparición de malos hábitos, derivados de la compensación material que los padres/madres ejercen sobre su hijo/a para paliar la ausencia. Sin embargo, las abuelas en las entrevistas no hacen alusión a éstos, aunque sí hacen hincapié en que lo material no reemplaza la presencia materna-paterna, tanto en su papel afectivo como de autoridad. 


\subsection{Las vidas personales de las abuelas y sus sentimientos frente al ejercicio de cuidado}

Atendiendo a los cambios ocurridos en el hogar tras la emigración, en las entrevistas salen a la luz los sentimientos derivados de la ausencia de la personas migrante. Las narraciones de las abuelas muestran una persistente alusión a los aspectos emocionales experimentados por las tres partes y relacionados con la tristeza: la persona migrante, sus ascendientes y sus descendientes. Se trata de una tristeza reflejada en palabras como "nostalgia, anhelo, extrañar, llorar y pesar"; o en el uso de expresiones como "sentirse aburrido, sentirse maluco, me hace mucha falta, o es muy duro".

La tristeza experimentada por las propias abuelas se verbaliza en relación a su rol de madres que ven a sus hijas/os emigrar. No parece que ellas tengan sentimientos de rencor o de abandono hacía las y los hijos emigrados, puesto que se supone de naturaleza común desear lo mejor para sus descendientes, aunque esto conlleve la emigración y la consiguiente reorganización del cuidado dentro de la familia. Así, de manera análoga a lo teorizado por Gioconda Herrera (2012), la migración es vista como una forma de conseguir el anhelado progreso $y$, por tanto, las emociones negativas como la tristeza producida por la ausencia y la distancia, se van racionalizando en sentimientos de resignación y conformidad, justificados por el deseo de ver realizadas a sus hijas e hijos y por el deseo de un futuro mejor que sirva para mantener a la familia.

Además es muy común expresar un sentimiento de dejar en libertad a las hijas para decidir sobre sus propias vidas, de no "cortarles las alas". De hecho, en las entrevistas salen a la luz casos de ruptura de las relaciones de pareja de la emigrante por cuestiones de maltrato o de abandono del hogar por parte de sus compañeros que ocurren en edad temprana de las criaturas. Estas situaciones generan en las abuelas un sentimiento de solidaridad femenina e inter-generacional hacia las hijas que necesitan de su apoyo emocional y logístico en sus labores de madres solteras, aunque esto conlleve en muchos casos, como argumentan Cecilia Salazar, Elisabeth Jiménez y Fernanda Wanderley (2010), agotamiento físico y empeoramiento de sus condiciones de vida.

A través del análisis de sus relatos, se percibe que la aceptación del cuidado de los menores ha sido en ocasiones una opción más impuesta que elegida, hecho que se puede interpretar como consecuencia de las lógicas de poder y los mandatos de género dentro de la familia (Izquierdo, 2003; Vega Solís, 2009). En consecuencia, se generan sentimientos negativos que no siempre son expresados abiertamente, 
sino que salen a la luz cuando se pregunta por las transformaciones que han sufrido sus vidas cotidianas, y quedan latentes en cambio cuando hablan de cómo fue tomada la decisión de que fuera ella y no otra persona la que ejerciera el cuidado o cuando se trata el tema de la toma de decisión de la persona emigrada de salir del país. En un primer análisis se podría afirmar que la aceptación de la situación emigratoria y los cambios que ello conllevaba fue positiva tanto para los menores como para las abuelas. Sin embargo, al profundizar en los sentimientos con respecto a sus vidas personales al margen de su rol de cuidadoras, se traslucen ciertas recriminaciones y deseos frustrados, quedando así manifiesta la experimentación de sentimientos ambivalentes:

\begin{abstract}
Los niños están a cargo mío (...). Es un problema porque ellos [los emigrados] planean irse y todo pero uno queda encartado y amarrado (...). Yo antes de tener un niño no permanecía en la casa, yo permanecía paseando, andando, de una parte y la otra, ahora no puedo (...). Yo prácticamente venía aquí a dormir no más, en cambio ahora sí estoy amarrada (E4: abuela Lorena, Pereira).
\end{abstract}

Los cambios en la cotidianeidad que se ponen de manifiesto en los relatos son de dos tipos principalmente. En primer lugar, en sentido negativo, referidos a la incapacidad para continuar con sus actividades de ocio y/o laborales; $y$, en segundo lugar, en sentido positivo, referido al hecho de que su situación económica ha mejorado y/o al cuidado y cariño recibido por parte de sus nieta/os. A pesar de que muchas de las abuelas entrevistadas ya ejercían el cuidado de sus nietos/as previamente a la migración, el desempeño del rol y la responsabilidad asumida era parcial, lo cual suponía también una limitación parcial. La alusión a sentirse "amarrada" se produce en reiteradas ocasiones como una expresión más de los cambios que ha producido la emigración en su vida personal. Estos cambios derivan en un hartazgo del cuidado de los/as nietos/as, producto de una extensión a lo largo de muchos años de esta situación familiar, prevista inicialmente sólo por un periodo de tiempo corto. Como muchas abuelas aluden, los emigrados se iban para dos o tres años, pero esos planes no se han cumplido. Se expresa un deseo de "volver a su vida" dado que las entrevistadas "ya cuidaron de sus propios hijos" y ahora no les corresponde desempeñar ese papel con sus nietos/as. En este sentido, Jeanine Anderson (2006) hace hincapié en que en muchos casos la "maquinaria de cooperación familiar" puede llegar al colapso, puesto que, algunas de las abuelas más que encajar con el papel de cuidadoras son ellas mismas las demandantes de cuidados. 
Los sentimientos negativos se relacionan también en parte con la sobrerresponsabilidad asumida por las abuelas. Dicha sobrerresponsabilidad se vincula en las entrevistas a tres causas. En primer lugar, en relación a la edad de las y los nietos, puesto que existe una mención a la creciente responsabilidad que supone el cuidado de adolescentes, con los peligros y temores que esta etapa conlleva. En segundo lugar, en relación a la edad de la cuidadora, puesto que, con el avance de la vejez y el empeoramiento del estado físico y mental, se presentan mayores dificultades para seguir desempeñando su papel. Y, por último, respecto a un deseo de querer disfrutar de una vida tranquila y sosegada, propia de su momento vital, la vejez. A pesar de aceptar las circunstancias de la situación emigratoria, las abuelas reclaman su propia vida y mayores cotas de autonomía y libertad. Estos sentimientos negativos frente al cuidado de los/as nietos/as no son visibles en un primer momento pero resultan cruciales a la hora de entender los efectos que la emigración de los padres-madres tiene, no sólo en los/as hijos/as que se quedan en origen, sino también sobre otras personas que hacen posible esta situación, como es el caso de las abuelas cuidadoras (Bianchi-Pernasilici et al., 2015).

Como hemos explicado, las entrevistadas, en su papel como cuidadoras, muestran un apego emocional nada desdeñable hacia sus nietos y nietas. Estos sentimientos, sin embargo, conviven a menudo con la expresión del deseo de finalizar con las tareas de cuidado que les han sido asignadas. Por tanto, existe una tensión - de nuevo una ambivalencia - entre, por un lado, la dependencia emocional del cariño tanto hacia sus hijos/as como hacia sus nietos/as y, por otro, el hartazgo y la sobrerresponsabilidad percibida respecto a dicho rol. Es una constante por parte de las abuelas, la consideración del cuidado de sus nietos/as como una responsabilidad que provoca cansancio y que, en algunos casos limita su autonomía y libertad, generando frustración.

\section{CONCLUSIONES}

El propósito de este artículo ha sido llevar a cabo una aproximación a las vivencias y reflexiones de las abuelas colombianas que ejercen el papel de cuidadoras principales de sus nietas/os en origen tras la emigración de la madre/padre de las criaturas. Las dos hipótesis desde las cuales partimos son: (1) las abuelas desempeñan un papel fundamental en el proceso de transnacionalización de los cuidados y su consecuente reorganización en el seno de los hogares tras la migración; y (2) la emigración de sus hijas e hijos influye de manera ambivalente en las experiencias vitales de las abuelas, en sus relaciones familiares, sus emociones y sentimientos. 
En relación a la primera hipótesis, para aproximarnos al punto de vista de las abuelas, hemos decidido tomar en cuenta la valoración que ellas hacen sobre la migración en general, para entender a grandes rasgos cuáles son los efectos del proceso migratorio familiar. En este sentido se evidencia la tensión que se genera entre los beneficios económicos obtenidos por el envío de remesas y los costes emocionales derivados de la pérdida de la afectividad cotidiana con la persona migrante. Esta tensión tiene características análogas a la que se produce a nivel global y que las economistas feministas la explicitan como la tensión entre la "sostenibilidad de la vida" y la "acumulación de capital" (Pérez Orozco y Lafuente, 2013). Esta ambivalencia en la valoración es acentuada por la situación de recesión económica en los países de destino de las migraciones, en este caso de España. De hecho, el trabajo de campo realizado a principios del año 2012 mostró las consecuencias de la crisis en el discurso de las abuelas-cuidadoras que, a menudo, representan los eslabones más débiles de las "cadenas globales de cuidados" (Molano Mijangos et al., 2012). Ellas expresan los efectos de la crisis económica española sobre el microcosmos de las familias en origen, en concreto sobre la disminución parcial o la falta total de las remesas económicas que durante años recibían.

Tras la emigración, la reorganización del cuidado y la restructuración familiar en torno a las y los hijos de las personas migrantes, se produce frecuentemente bajo patrones de continuidad, determinados a su vez por patrones de género. Como se ha destacado, dicha continuidad en el desempeño de las tareas de cuidado puede incluso llegar a ejercer un papel relevante en la decisión de emigrar, considerando ésta como una condición que posibilita la salida del país de origen de la madre/padre sin cambios bruscos en la organización familiar. Son frecuentes los casos en los que la madre (o suegra) de la persona emigrante ejercía labores de cuidado de las y los hijos, evidenciando así que la conciliación de la vida laboral, personal y familiar está presente antes de la migración. Dicha continuidad está basada en el rol social de la mujer como cuidadora, que ante la emigración se traslada de la madre a la abuela, mostrando la ausencia de los hombres en este ámbito, algo ampliamente demostrado por los estudios feministas y de genero.

En definitiva, es posible afirmar, como planteábamos en la primera hipótesis que conduce este texto, que las abuelas cumplen con un papel fundamental dentro del desarrollo del proceso migratorio y su consecuente reorganización del cuidado familiar. Además, se evidencia que el contexto de crisis económica en España afecta de manera transnacional a los hogares en origen. 
En relación a la segunda hipótesis, que atiende a la esfera emocional, se constatan, por una parte, sentimientos de tristeza derivada de la ausencia de la persona migrante pero, por otra, el ejercicio del cuidado asumido por las abuelas puede llegar a desencadenar, en algunos casos, ganancias de tipo afectivo a través de relaciones de cuidado bidireccionales. Si se dirige la mirada hacia las relaciones interpersonales dentro de la familia, las percepciones son ambivalentes. Por un lado, se explicita un buen mantenimiento de las relaciones afectivas gracias a las frecuentes comunicaciones por teléfono $e$ internet, y a la preocupación del día a día por parte de los padres-madres por las vivencias de sus hijos/as y de la vida familiar en su conjunto. Pero, por otro lado, encontramos también un reconocimiento de la distancia existente entre los modos de vida de origen y destino, y de los límites que la presencia virtual tiene para el desarrollo de unas relaciones interpersonales afectivas y más cotidianas. Además, al igual que aparece reflejado en otras investigaciones (Herrera y Carrillo, 2009; González y Restrepo, 2010), la afectividad es uno de los elementos esenciales del ejercicio de cuidado $y$, en este sentido, en los casos estudiados, se detectan relaciones de cuidados mutuos y bidireccionales entre las abuelas y sus nietos/as. En relación a las reflexiones de las abuelas sobre su propia situación, la asunción del rol de cuidadora dentro de la familia supone para ellas una vuelta al pasado como responsables de la reproducción social. Esto implica volver de nuevo a un ciclo de vida doméstico que ellas creyeron finalizado con la llegada a la edad adulta de sus hijos e hijas, pero esta vez con la singularidad de que se convierte en una 'adopción temporal' (Salazar et al., 2010) y que puede ser finalizada a petición de la madre o del padre emigrado con la reagrupación familiar.

En resumen, la segunda hipótesis que guiaba este texto es pertinente puesto que se detectan claramente ambivalencias en las percepciones sobre los efectos del proceso migratorio en la vida de las abuelas, determinadas por la tensión entre el rol social de madre-abuela y sus propias necesidades (Merton, 1980). Por un lado, se evidencia el papel activo y de complicidad de las abuelas a la hora de ayudar a sus hijas pero, por otro lado, se destaca la resistencia de éstas a los mandatos de género, expresada en forma de quejas, poniendo en cuestión el rol de cuidadora, que brinda amor y atenciones de manera incondicional, solamente por el hecho de ser la abuela. Dicho esto, merece la pena aclarar que este proceso no es exclusivo del fenómeno migratorio, sino que forma parte de los efectos que la difícil conciliación de la vida familiar y laboral tiene sobre la organización del cuidado a nivel mundial (Pérez Orozco, 2006). La delegación del cuidado se produce también en 
las sociedades de destino con las abuelas autóctonas que, a menudo, se ven obligadas a retomar su rol de cuidadoras dadas las difíciles condiciones de conciliación de las familias (Pérez Ortiz, 2006; Villalba Quesada, 2002).

La reorganización de las relaciones de cuidados y la multiplicidad de deseos de cada uno de los miembros de la familia sobre las formas de ejercerlos genera una cotidianidad difícil de gestionar que ha sido explorada más desde el lado de las mujeres migrantes, que desde el de las cuidadoras en origen. El estudio evidencia la existencia de sentimientos complejos y ambivalentes que afectan a las abuelas cuidadoras en origen, y que deben ser contemplados con mayor intensidad desde los análisis sociológicos de cara a incrementar y profundizar la comprensión del fenómeno migratorio y sus consecuencias, así como también en pos de mejorar la intervención social y política sobre dichos efectos.

\section{BIBLIOGRAFÍA}

Aguirre, R., García Saínz, C., Carrasco, C., 2005, El tiempo, los tiempos, una vara de desigualdad, CEPAL, Santiago de Chile.

Anderson, B., 2000, Doing the dirty work? The global politics of domestic labour, Zed Books, Londres.

Anderson, J., 2007, "Género de Cuidados", en M. Barrig (Ed.), Fronteras interiores. Identidad, diferencia y protagonismo de las mujeres, IEP, Lima, pp. 71-93.

Ariza, M., 2007, “Itinerario de los estudios de género y migración en México", en M. Ariza y A. Portes (Eds.), El país transnacional. Migración mexicana, cambio social a través de la frontera, UNAM, Instituto de Investigaciones Sociales, México, pp. 453-512.

Arteaga, A.M., 2010, Mujeres migrantes andinas. Contexto, políticas y gestión, Unión Europea y Oxfam Gran Bretaña, Santiago de Chile.

Bericat Alastuey, E., 2000, “La sociología de la emoción y la emoción en la sociología", en Papers, vol. 62, pp. 145-176.

Baldassar, L., Merla, L., 2014, “Locating transnational care circulation in migration and family studies", en L. Baldassar y L. Merla (Eds.), Transnational families, migration and the circulation of care, Routledge, Londres, pp. 25-60. 
Bianchi-Pernasilici, G.M., 2014, Migraciones y trabajo doméstico-de cuidados. El caso de la población andina en el Gran Bilbao, Tesis doctoral, Universidad del País Vasco, Leioa.

Bianchi-Pernasilici, G.M., González-Rábago, Y., Piras, G., 2015, "Enfrentando la transnacionalización del cuidado: abuelas cuidadoras en un contexto de alta migración", en Revista Internacional del Estudios Migratorios, vol. 5(1), pp. 31-60.

Blanco, C., 1993, La integración de los inmigrantes en las sociedades receptoras: método de análisis y aplicación al País Vasco, Tesis doctoral, Universidad de Deusto, Bilbao.

Blanco, C., 2007, "Transnacionalismo. Emergencia y fundamentos de una nueva perspectiva migratoria", en Papers, vol. 85, pp. 13-29.

Bryceson, D., Vuorela, U., 2002, The transnational family: new European frontiers and global networks, Berg, Oxford/New York.

Carrasco, C., Borderías, C., Torns, T., 2011, "Introducción. El trabajo de cuidados: antecedentes históricos y debates actuales", en C. Carrasco, C. Borderías, y T. Torns (Eds.), El trabajo de cuidados. Historia, teoria y políticas, Catarata, Madrid, pp. 13-96.

Carrasquer, P., 2013, “El redescubrimiento del trabajo de cuidados: algunas reflexiones desde la sociología", en Cuadernos de Relaciones Laborales, vol. 31 (1), pp. 91-113.

Cerulo, M., 2009, II sentire controverso. Introduzione alla sociolgía delle emozioni, Carocci editore, Roma.

Chant, S.H., 1992, Gender and migration in developing countries, Belhaven Press, London.

Colectivo IOÉ, 1999, Inmigrantes, trabajadores, ciudadanos: una visión de las migraciones desde España, Universitat de València, Valencia.

Colectivo IOÉ, 2012, Impactos de la crisis sobre la población inmigrante, OIM, Madrid.

Durán, M.A., 2011, "El trabajo del cuidado en el marco macroeconómico", en M.A. Durán (Ed.), El trabajo del cuidado en América Latina y España, Fundación Carolina CEALCl, Madrid, pp. 11-32.

Delgado Wise, R., Covarrubias, H., Rodríguez, H., 2004, "Organizaciones transnacionales de migrantes y desarrollo regional en Zacatecas", en Migraciones Internacionales, vol. 4 (2), pp. 159-181.

Echeverri Buriticá, M., 2014, "A los dos lados del atlántico. Reconfiguraciones de los proyectos migratorios y la vida familiar 
transnacional de la población colombiana en España", en Papeles del CEIC, vol. 2014/2, n 109, CEIC (Centro de Estudios sobre la Identidad Colectiva), Universidad del País Vasco.

Escrivá, A., 2004, Securing care and welfare of dependents transnationally: Peruvians and Spaniards in Spain, Working Paper $n^{\circ} 404$, Oxford Institute of Ageing, Oxford.

Gálvez, L., Torres, J., 2010, Desiguales: Mujeres y hombres en la crisis financiera, Icaria, Barcelona.

Garay Salamanca, L.J., Rodríguez Castillo, A., 2005, Estudio sobre migración internacional y remesas en Colombia, OIM, Bogotá.

Gil Araújo, S., 2005, "Cartografías Migratorias: Migraciones internacionales en el marco de las relaciones Norte-Sur", en N. Zúñiga García-Falces (Coord.), La migración, un camino entre el desarrollo y la cooperación, Centro de Investigación para la PazFUHEM, Madrid, pp. 13-52.

González, L.A., Restrepo, J.E., 2010, "Prácticas de continuidad de los vínculos parentales en las familias transnacionales colombianas en España", en Revista Latinoamericana de Estudios de Familia, vol. 2, pp. 79-97.

Herrera, G., 2012, "Repensar el cuidado a través de la migración internacional: mercado laboral, estado y familias transnacionales en Ecuador", en Cuadernos de Relaciones Laborales vol. 1, pp. 139159.

Herrera, G., 2013, Lejos de tus pupilas: familias transnacionales, cuidados y desigualdad social en Ecuador, FLACSO-Ecuador, Quito.

Herrera, G., Carrillo, M.C., 2009, "Transformaciones familiares en la experiencia migratoria ecuatoriana. Una mirada desde los contextos de salida", en Revue des Mélanges de la Casa Velásquez, vol. 39-1, pp. 97-114.

Hochschild, A., 1975, "The Sociology of Feeling and Emotion: Selected Possibilities", en M. Millman y R.M. Kanter (Eds.), Another Voice. Feminist perspectives on social life and social science, Anchor Books, Nueva York, pp. 280-307.

Hochschild, A., 2001, "Las cadenas mundiales de afecto y asistencia y la plusvalía emocional", en A. Giddens y W. Hutton (Eds.), El límite. La vida en el capitalismo global, Tusquets Editores, Barcelona, pp. 187-207. 
Hondagneu-Sotelo, P., Avila, E., 1997, "I'm here, but I'm there: The meanings of Latina transnational motherhood", en Gender and Society, vol. 2 (5), pp. 548-71.

Hondagneu-Sotelo, P., 2007, "La incorporación del género a la migración: 'No sólo para feministas', ni sólo para la familia", en M. Ariza y A. Portes (Eds.), El país transnacional. Migración mexicana, cambio social a través de la frontera, UNAM, Instituto de Investigaciones Sociales, México, pp. 423-452.

Hughes, C., 2002, Key Concepts in Feminist Theory and Research, Sage Publications, London.

Izquierdo, M.J., 2003, "Del sexismo y la mercantilización del cuidado a su socialización: hacia una política democrática del cuidado", ponencia presentada en el congreso Cuidar cuesta: costes y beneficios del cuidado, Emakunde, Donosti, pp. 121-154.

Lagomarsino, F., 2014, “Familias en movimiento: más allá de los estereotipos de la maternidad transnacional", en Papeles del CEIC, vol. 2014/2, n 108, CEIC (Centro de Estudios sobre la Identidad Colectiva), Universidad del País Vasco.

Levitt, P., DeWind, J., Vertovec, S., 2003, "International perspectives on transnational migration: an introduction", en International Migration Review, vol. 37 (3), pp. 565-575.

MacInnes, J., Pérez Díaz, J., 2008, “La tercera revolución de la modernidad: la revolución reproductiva", en REIS. Revista Española de Investigaciones Sociológicas, vol. 122, pp. 89-118.

Marroni, M. G., 2000, "Él siempre me ha dejado con los chiquitos y se ha llevado a los grandes... ajustes y desajustes familiares de la migración", en D. Barrera Bassols y C. Oechmichen Bazán (Eds.), Migración y relaciones de género en México, GIMTRAP/UNAM, México, pp. 87-117.

Martínez Franzoni, J., 2008, Domesticar la incertidumbre en América latina. Mercado laboral, política social y familias, Universidad de Costa Rica, San José.

Mauss, M., 2002, Saggio sul dono. Forma e motivo dello scambio nelle societá arcaiche, Einaudi, Torino.

Mejía, W., Ortiz, D., Puerta, C., Mena, J., Díaz, E., 2009, Encuesta nacional 2008-2009. Resultados generales de migraciones internacionales y remesas, Fundación Esperanza, Bogotá. 
Merton, R. K., 1980, Ambivalencia sociológica y otros ensayos, EspasaCalpe, Madrid.

Molano Mijangos, A., Robert, E., Domínguez, M.G., 2012, Cadenas globales de cuidados: sintesis de resultados de nueve estudios en América Latina y España, ONU Mujeres, Santo Domingo.

Ostergaard-Nielsen, E., 2003, “The politics of migrants' transnational political practices", en International Migration Review, vol. 3 (37), pp. 760-786.

Parella, S., 2000, "El trasvase de desigualdades de clase y etnia entre mujeres: los servicios de proximidad", en Papers, vol. 60, pp. 275289.

Parreñas, R., 2001, Servants of globalization: Women, migration, and domestic work. Stanford University Press, California.

Pedone, C., 2006, “'Tu siempre jalas a los tuyos'. Cadenas y redes migratorias de las familias ecuatorianas hacia España", en G. Herrera (Ed.), La migración ecuatoriana: transnacionalismo, redes e identidades, FLACSO-Ecuador, Quito, pp. 105-144.

Pérez Orozco, A., 2006, Perspectivas feministas en torno a la economía: el caso de los cuidados, Consejo Económico y Social, Madrid

Pérez Orozco, A., Lafuente, S., 2013, “Economía y (trans)feminismo retazos de un encuentro", en M. Solá y E. Urko (Eds.), Transfeminismos. Epistemes, fricciones y flujos, Txalaparta, Orkoien-Navarra, pp. 91-108.

Pérez Ortiz, L., 2006, Las abuelas como recurso de conciliación entre la vida familiar y laboral. Presente y futuro, Ministerio de Trabajo y Asuntos Sociales, Instituto de la Mujer, Madrid.

Puyana, Y., 2009, Entre aquí y allá. Las familias transnacionales colombianas, Universidad Nacional de Colombia y Fundación Esperanza, Bogotá.

Salazar, C., E. Jiménez, F. Wanderley, 2010, Migración, cuidado y sostenibilidad de la vida, CIDES-UMSA, Bolivia.

Sayad, A., 2002, La doppia assenza, Raffaello Cortina editore, Milano.

Sassen, S., 2003, Contrageografías de la globalización. Género y ciudadanía en los circuitos transfronterizos, Traficantes de Sueños, Madrid. 
Sørensen, N., 2008, "La familia transnacional de latinoamericanos/as en Europa", en G. Herrera y J. Ramírez (Eds.), América Latina migrante: Estado, familias, identidades, FLACSO-Ecuador, Quito, pp. 259-279.

Vega Solís, C., 2009, Culturas del cuidado en transición. Espacios, sujetos $e$ imaginarios en una sociedad de migración, Editorial UOC, Barcelona.

Villalba Quesada, C., 2002, Abuelas cuidadoras, Tirant lo Blanch, Valencia.

Wagner, H., 2008, "Maternidad transnacional: discursos, estereotipos, practicas", en G. Herrera y J. Ramírez (Eds.), América latina migrante: estado, familia, identidades, FLACSO-Ecuador, Quito, pp. 325-341.

Yeates, N., 2005, "Global care chains: a critical introduction", en Global Migration Perspective, vol. 44, Global Commission on International Migration, Geneva.

Zimmerman, M.K., Litt, J.S., Bose, C.E., 2006, "Globalization and multiple crises of care", en M.K. Zimmerman, J.S. Litt y C.E. Bose (Eds.), Global dimensions of gender and care work, Stanford University Press, California. 